\title{
A case report of sirenomelia with two umbilical arteries: an unusual finding in a rare case
}

\author{
Swati Trivedi ${ }^{1 *}$, Oby Nagar $^{2}$, J. P. Soni ${ }^{3}$, Shashank Trivedi ${ }^{4}$, Prasoon Rastogi ${ }^{5}$
}

\author{
${ }^{1}$ Department of Obstetrics and Gynaecology, ${ }^{3}$ Department of Paediatrics, Dr. S N Medical College, Jodhpur, Rajasthan, \\ India \\ ${ }^{2}$ Department of Obstetrics and Gynaecology, S.M.S. Medical College, Jaipur, Rajasthan, India \\ ${ }^{4}$ Department of Pediatrics, Guru Gobind Singh Government Hospital, New Delhi, India \\ ${ }^{5}$ Department of Endocrinology, All India Institute of Medical Sciences, Jodhpur, Rajasthan, India
}

Received: 15 July 2021

Accepted: 04 August 2021

\author{
*Correspondence: \\ Dr. Swati Trivedi, \\ E-mail: krishnaagnihotri.kgmc@gmail.com
}

Copyright: (c) the author(s), publisher and licensee Medip Academy. This is an open-access article distributed under the terms of the Creative Commons Attribution Non-Commercial License, which permits unrestricted non-commercial use, distribution, and reproduction in any medium, provided the original work is properly cited.

\begin{abstract}
Sirenomelia also known as Mermaid syndrome is a rarely encountered fusion anomaly of the caudal region of body often associated with Potter's facies, single umbilical artery and various visceral abnormalities which make it irreconcilable with life. Here we report a case of sirenomelia delivered in our tertiary care hospital by lower segment cesarean section to a 24 year old third gravida with no previous live issues. No high risk factors could be identified with the mother including diabetes mellitus. Baby was born alive with Potter's facies. Both the lower limbs were merging into each other like a mermaid's tail (hence called mermaid syndrome). Sex of the baby could not be identified and the urogenital and anal orifices were absent. Umbilical cord stump had two umbilical arteries. The baby died after 20 hours of life. There appears to be no definitive modality for diagnosing sirenomelia in the antenatal period. However, if a patient has consistently low AFI without any history of leaking per vaginum, high resolution USG or colour Doppler should be done at the earliest to look for the cause.
\end{abstract}

Keywords: Fusion anomaly, Mermaid syndrome, Perinatal outcome, Potter's facies, Sirenomelia, umbilical arteries

\section{INTRODUCTION}

Sirenomelia or Mermaid syndrome is a rare and fatal developmental defect of lower body, characterized by apparent fusion of lower limbs in various degrees into a single limb, giving the appearance of a mermaid's tail.

Sirenomelia phenotype has been linked to Greek mythology, where Sirens were mentioned as dangerous creatures with head of a woman and body of a bird, that over the years evolved as mermaid-like brute. The incidence of sirenomelia varies from 0.8 to 1 case per $1,00,000$ births. ${ }^{1}$ So far very few such cases have been reported in literature. We intend to report our case for its rarity and presence of two umbilical arteries.

\section{CASE REPORT}

Mrs. AB 24-year pregnant female was admitted to Umaid hospital at 37-week gestational age on account of oligohydramnios, breech presentation with decreased fetal movements and no previous live issues.

She was G3P (2) with previous term vaginal deliveries: first was an IUD(cause unknown) and second died after 1 month of life due to fever/pneumonia. Patient had history of secondary infertility, for which ovulation induction with Clomiphene citrate was done. She came to our hospital as an unbooked case, but was on follow-up elsewhere since 5th month of pregnancy (patient had lost follow-up after conception due COVID pandemic). All antenatal 
biochemical investigations were within normal limits. Two transabdominal sonography were done during the period of follow-up at 21 and 32 week; the finding common was a consistently low AFI (1-2) with no fetal malformations reported.

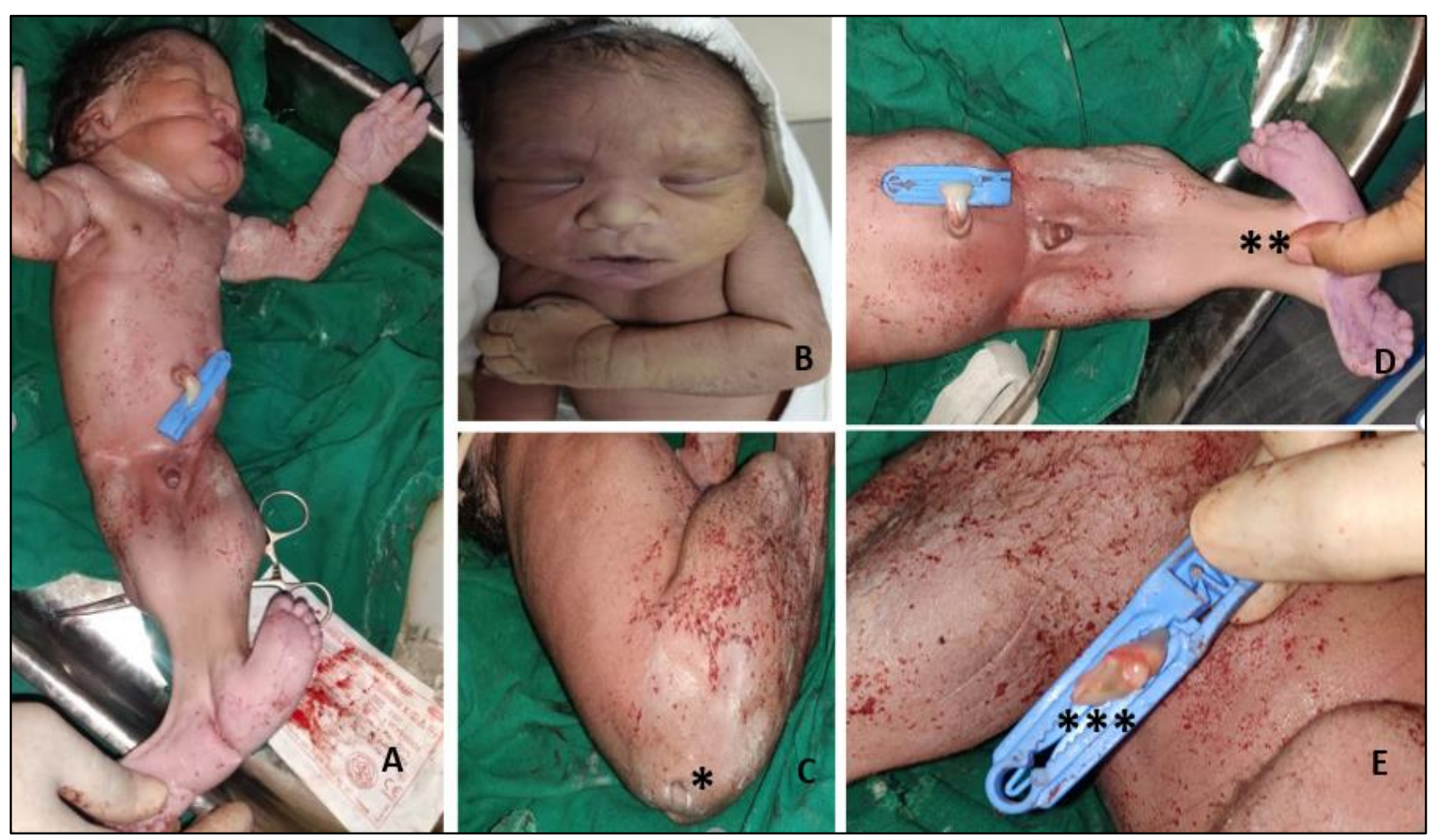

Figure 1: (A) Gross morphology (B) Potter's facies (C) Anal dimple (*) (D) Merged lower limbs-mermaid tail (**). (E) Umbilical cord (***2 Umbilical arteries + 1 Umbilical vein).
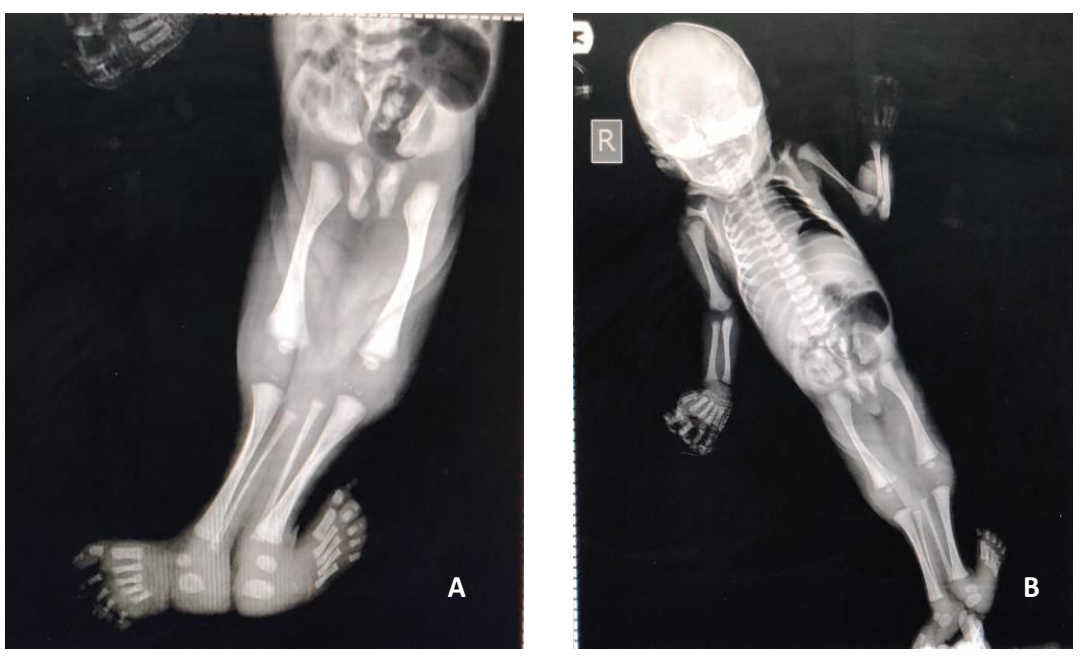

Figure 2: (A) Distinct lower limb $x$ ray AP view. (B) Whole body $x$ ray AP view.

On admission, patient's vitals were normal, fundal height corresponding to 32-34 week with uterus full of fetus. She was taken for emergency caesarean section in view of oligohydramnios with breech presentation and fetal distress. A $2.0 \mathrm{~kg}$ baby with fused lower limbs (Figure 1A) was delivered and scanty amniotic fluid drained. The baby was limp on extraction with feeble cry, grunting and peripheral cyanosis. APGAR score at $1 \mathrm{~min}, 5 \mathrm{~min}$ and 10 min were 3/10, 4/10 and 4/10 respectively. Baby was kept on high flow oxygen but died 20 hours after birth due to respiratory distress. No urine or stool were passed.

On physical examination, baby had dysmorphic appearance with hypertelorism, prominent epicanthic fold, 
low set malformed ears, broad nasal bridge with downward curved nose suggestive of Potter's facies (Figure 1B) along with short neck, narrow chest depicting lung hypoplasia, ambiguous genitalia with small penis and absent urogenital and anal orifices (Figure 1C), bilaterally fused non-rotated lower limb with externally rotated feet (Figure 1D). The umbilical cord stump showed two umbilical arteries and one vein (Figure 1E).

X-ray skeletal survey revealed bilateral femur, tibia, fibula with foot bones but sacral agenesis. Thus, our case is Type I Sirenomelia according to Stocker and Heifetz classification of sirenomelic infants (Figure 2A). The bowel loops appear dilated with air bubble. Pneumothorax can be seen on left side of the chest with hypoplastic right lung (Figure 2B).

Ultrasonography revealed bilateral small kidneys with empty urinary bladder. Rectal and Anal agenesis/ atresia was associated with proximal bowel dilatation. There was no scrotum but undescended testes were seen.

Autopsy and genetic workup could not be done as the parents did not give consent.

There was no history of tobacco or alcohol consumption, diabetes mellitus, radiation exposure in early pregnancy or ingestion of teratogenic drugs, any genetic or congenital anomaly in previous born babies or any such running in the family. Mother's intraoperative and postoperative periods were uneventful.

\section{DISCUSSION}

Sirenomelia was first described in 16th century by Rocheus and Polfyn. The diagnosis, which is obvious at birth, can be made antenatally with high resolution ultrasonography or colour doppler as early as 13 weeks (oligohydramnios, renal agenesis, fused femur, decreased distance between femurs and absent mobility of lower limbs). Apart from the striking phenotype which includes fusion of lower limbs into a single limb, sirenomelia is a multisystem condition associated with severe visceral abnormalities, most commonly urogenital and gastrointestinal which makes it incompatible with life; death usually ensues in perinatal period. The facial abnormality usually found, known as Potter's facies includes: large, low-set ears, prominent epicanthic fold, hypertelorism, flat nose and receding chin. Potter's facies with oligohydramnios and pulmonary hypoplasia is known as Potter's syndrome.

According to the presence of skeletal elements in the thigh and leg, sirenomelia has been classified by Stocker and Heifetz into 7 types. In type I, the mildest form, all bones are present, and fusion affects only superficial tissues. In type VII, the most severe form, only a single bone is present, with absent legs or feet. ${ }^{2}$
The primary molecular defect underlying sirenomelia remains unknown. Two main pathogenic hypotheses proposed by clinical studies to explain the causal mechanism are the vascular steal hypothesis and the defective blastogenesis hypothesis. The vascular steal hypothesis suggests that the SUA of vitelline origin diverts blood to the placenta, leaving the lower body with a severely deficient circulation and nutrient supply resulting in agenesis of midline structures along with abnormal approximation of lower limbs. Defective blastogenesis hypothesis, initially shaped in 19th century, states that primary defect of blastogenesis occurs during the final stages of gastrulation at tailbud stage, corresponding to third gestational week.

The presence of single umbilical artery (SUA) of vitelline origin has been considered as characteristic or even pathognomonic of sirenomelia and was proposed to be used for the differential diagnosis of other malformations of lower body such as caudal dysgenesis. Occasionally cases of sirenomelia may present with two symmetrical umbilical arteries, though they are of abnormal origin. In our case the baby also had two umbilical arteries, thus challenging the vascular steal hypothesis of Sirenomelia.

This syndrome has strong association with maternal Diabetes mellitus. Intake of tobacco antenatally, cadmium, lead, vitamin A, nutritional deficiency and vascular hypoperfusion are proposed teratogen for sirenomelia. ${ }^{3}$ Since none of these factors were present in our patient, the cause of sirenomelia remains eluded here.

Experimental data suggest that sirenomelia has a genetic basis resulting from a defect in RAR or Bmp signalling in the caudal embryonic region. ${ }^{4}$

\section{CONCLUSION}

Sirenomelia is a rare and lethal condition with poor prognosis. Considering the current standards of care and lack of availability of appropriate modalities at grassroot level, diagnosing Sirenomelia in the antenatal period seems a real challenge. However, on the basis of clinical suspicion in a patient with consistently low AFI without any history of leaking per vaginum, high resolution USG or colour Doppler should be done at the earliest to identify the cause. The anomaly if diagnosed antenatally, we can atleast prevent maternal morbidity by holding back caesarean section for fetal indication (as we know the neonatal survival is close to nil) and giving a trial for vaginal delivery. Also, we can offer termination of pregnancy at an early gestation, thereby preventing mother from the burden of carrying an anomalous pregnancy to term. Sirenomelia is strongly associated with single umbilical artery and diabetes mellitus in mother but in our case no such association was found.

Funding: No funding sources

Conflict of interest: None declared

Ethical approval: Not required 


\section{REFERENCES}

1. Jones KL, Jones MC, Campo M del. Smith's Recognizable Patterns of Human Malformation. 8th Edition Elsevier Health Sciences. 2013;1091.

2. Stocker JT, Heifetz SA. Sirenomelia. A morphological study of 33 cases and review of the literature. Perspect Pediatr Pathol. 1987;10:7-50.

3. Prasad S, Anupama J. Rare Case Report of Sirenomelia: The Mermaid Baby. Obstet Gynaecol. 2017;4(12):3.
4. Zakin L, Reversade B, Kuroda H, Lyons KM, De Robertis EM. Sirenomelia in Bmp7 and Tsg compound mutant mice: requirement for Bmp signaling in the development of ventral posterior mesoderm. Dev Camb Engl. 2005;132(10):2489-99.

Cite this article as: Trivedi S, Nagar O, Soni JP, Trivedi S, Rastogi P. A case report of sirenomelia with two umbilical arteries: an unusual finding in a rare case. Int J Reprod Contracept Obstet Gynecol 2021;10:3640-3. 